\title{
The problem of isotropic rectangular plate with four clamped edges
}

\author{
C ERDEM IMRAK* and ISMAIL GERDEMELI \\ Istanbul Technical University, Faculty of Mechanical Engineering, Mechanical \\ Engineering Department, Gümüşsuyu, TR-34437, Istanbul, Turkey \\ e-mail:imrak@itu.edu.tr
}

MS received 25 May 2006; revised 2 August 2006

\begin{abstract}
The examination of the exact solution of the governing equation of the rectangular plate is important for many reasons. This report discusses in exact solution of the governing equation of an isotropic rectangular plate with four clamped edges. A numerical method for clamped isotropic rectangular plate under distributed loads and an exact solution of the governing equation in terms of trigonometric and hyperbolic function are given. Finally, an illustrative example is given and the results are compared with those reported earlier. This method is found to be easier and effective. The results show reasonable agreement with other available results, but with a simpler and practical approach.
\end{abstract}

Keywords. Rectangular plate; clamped plate; uniform load; deflection.

\section{Introduction}

The deflection of rectangular plates with clamped at four edges and subject to the action of uniformly distributed loads is a problem that has received considerable attention because of its technical importance. This paper analyses the deflections of an isotropic rectangular clamped thin plates under uniformly distributed loads. A plate is called thin when its thickness is at least one order of magnitude smaller than the span of the plate. The bending and buckling of rectangular plates has been a subject of study in solid mechanics for more than a century. Many exact solutions for isotropic linear elastic thin plates have been developed; most of them can be found in Timoshenko's monographs (Timoshenko \& Woinowsky-Krieger, 1959). For clamped rectangular thin plates no accurate results appear to be available. Approximate solutions were also suggested, but these resulted in a notable loss of accuracy (Wang \& El-Sheikh, 2005). Many have calculated the deflections of rectangular plates with various supports using different methods Hencky (1913), Wojtaszak (1937), Timoshenko (1938), Evans (1939), Young (1940), Hutchinson (1992), Wang et al (2002), and Taylor \& Govindjee (2004). Some of them are approximate methods. Two main methods of approach have been used for obtaining the solution of the maximum deflection for clamped thin rectangular

${ }^{*}$ Corresponding author 
plates under uniform load; these are the double cosine series (Szilard, 1974) and the superposition method as a generalization of Hencky's solution (Evans 1939), Wojtaszak (1937), Young (1940). After the first numerical results for calculating deflections in clamped rectangular plates, the progress was made by Hencky (1913). Hencky's method is well known to converge quickly, but does pose some slightly tricky issues with regard to programming due to over/underflow problems in the evaluation of hyperbolic trigonometric functions. The first term in the series expansion dominates the series for the case of a square plate, but when the ratio of the side lengths increases this property disappears.

Hutchinson (1992) used the solution from which was presented in Timoshenko \& Woinowsky-Krieger (1959) and tabulated deflections for uniformly loaded rectangular plates. Obtaining the numerical values of deflections for a rectangular plate may be difficult (Imrak \& Gerdemeli, 2007). Evans has calculated the values of the center deflections for edges and centers various aspect ratios of the sides using Timoshenko's method. This method is based on the principle that the solution is obtained by combining the known solution of simply supported plate under uniform loading with that of a plate bent by moments distributed along the pairs of opposite edges. To obtain the values of maximum deflections for rectangular plates carrying uniform load is difficult (Erdogan \& Arpaci, 1990).

Authors in their recent study proposed a single cosine series for rectangular clamped plates. In this paper, a comprehensive method is presented for the numerical solution of the clamped rectangular plate problem under uniformly distributed loads and boundary conditions. The center deflection function of the fully clamped rectangular plate has three terms where the first one denotes the deflection function for the case of a strip and the others denote the effect due to the edges. The unknown three coefficients of the deflection function are determined by using the boundary conditions. A set of three linear equations depending upon the aspect ratios are given. The expansion of the center deflection function is given in a series form and the first term dominates the series. Therefore, it is shown that the series are rapidly convergent. An illustrative example is included of uniform load acting on a fully clamped rectangular plate. The calculation is performed for rectangular plate of uniform thickness. The result is compared with similar numerical techniques.

\section{Problem definition}

According to the classic theory of plate bending, a small deflection is defined as small compared to the plate thickness. The governing equation expressing the relationship between the rectangular plate lateral load and its deflection in the case of with small deflection is

$$
D \nabla^{4} w=D\left(\frac{\partial^{4} w}{\partial x^{4}}+2 \frac{\partial^{4} w}{\partial x^{2} \partial y^{2}}+\frac{\partial^{4} w}{\partial y^{4}}\right)=q
$$

where $w$ denotes the small deflections of the plate midsurface. $D=E h^{3} / 12\left(1-v^{2}\right)$ is the flexural rigidity of the plate cross-section, $q$ is a constant normal load per unit area, $\nabla^{4} \equiv \nabla^{2}\left(\nabla^{2}\right)$ denotes the bi-harmonic operator and $\nabla^{2}$ is Laplacian operator. Consider an isotropic rectangular plate of length $a$, width $b$, thickness $h$, modulus of elasticity $E$, and Poisson's ratio $v$ as depicted in figure 1 .

The problem of a rectangular plate clamped at four sides with a uniform load is considered. The sides of the rectangular section are at $x= \pm a$ and $y= \pm b$, and it is supposed throughout 


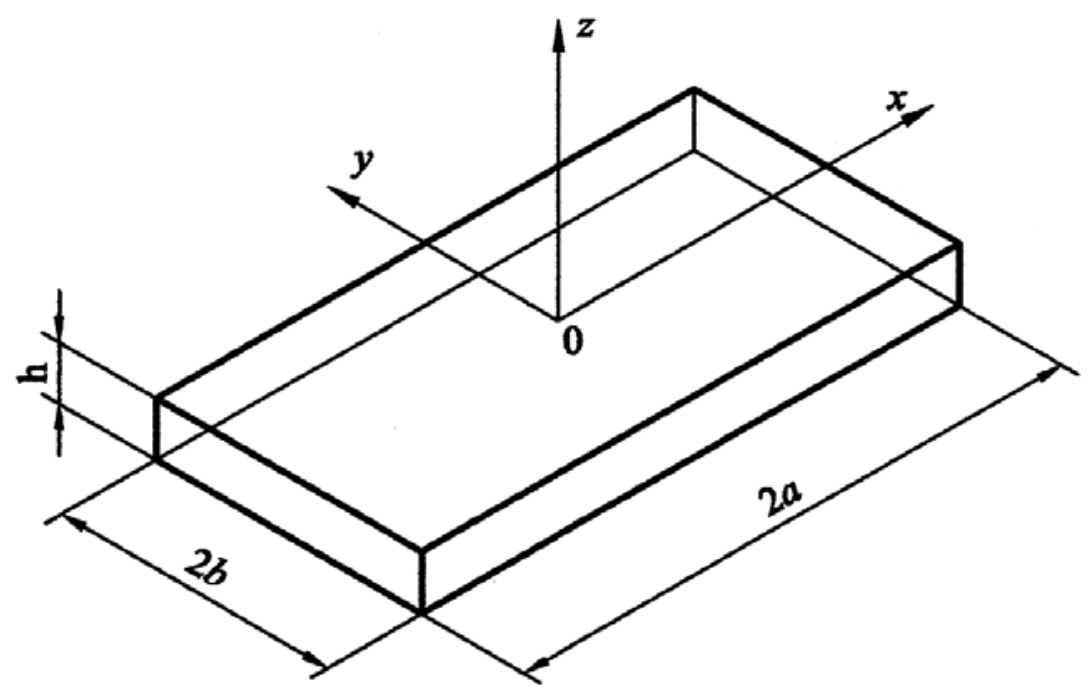

Figure 1. Coordinate system and dimensions for a thin rectangular plate.

the paper that $a \geq b$. The boundary conditions for the deflection function are

$$
\begin{aligned}
w & =0 \text { for } x= \pm a, \\
w & =0 \text { for } y= \pm b, \\
\frac{\partial w}{\partial x} & =0 \text { for } x= \pm a, \\
\frac{\partial w}{\partial y} & =0 \text { for } y= \pm b .
\end{aligned}
$$

An exact solution of the governing equation (1) subject to the boundary conditions (2) is given by Taylor \& Govindjee (2004). The problem of the simply supported plate with the uniform loading is solved first, giving the deflection function for the strip case and then a deflection function which shows the effects due to the edges is superimposed. Here we consider a method for efficiently determining a very large number of terms in the series. The series is given by

$$
\begin{aligned}
w(x, y)= & \frac{q b^{4}}{24 D}\left[\left(1-\frac{y^{2}}{b^{2}}\right)^{2}+\sum_{m=1,3 \ldots}^{\infty} \frac{\cos \frac{m \pi y}{2 b}}{\cosh \frac{m \pi a}{2 b}}\left(A_{m} \cosh \frac{m \pi x}{2 b}+B_{m} \frac{x}{a} \sinh \frac{m \pi x}{2 b}\right)\right. \\
& \left.+\sum_{m=1,3 \ldots}^{\infty} C_{m} \frac{\cos \frac{m \pi x}{2 a}}{b \cosh \frac{m \pi b}{2 a}}\left(y \sinh \frac{m \pi y}{2 a}-b \tanh \frac{m \pi b}{2 a} \cosh \frac{m \pi y}{2 a}\right)\right],
\end{aligned}
$$

where the first term gives the deflection function for the strip case in $-b \leq y \leq b$. The others show the effects due to the edges. The coefficients are then determined from the boundary conditions (2) that at the edges the slope is zero. One can obtain the following system of linear 
equations for determining the constants $A_{m}, B_{m}$ and $C_{m}$ :

$$
\begin{aligned}
& A_{m}+B_{m} \tanh \frac{m \pi a}{2 b}=\frac{2^{8}(-1)^{(m-1) / 2}}{\pi^{3}}\left[\frac{1}{m^{3}}-\frac{12}{\pi^{2} m^{5}}\right], \\
& A_{m} \frac{m(m-1) \pi}{4 b} \tanh \frac{m \pi a}{2 b}+B_{m}\left(\frac{b}{a} \tanh \frac{m \pi a}{2 b}+\frac{m \pi}{2}\right) \\
& \quad+\sum_{r=1,3 \ldots}^{\infty} C_{r} \frac{(-1)^{(m+r-2) / 2}}{8 r \pi\left(1+\frac{a^{2}}{b^{2}}\right)^{2}}=0, \\
& \sum_{r=1,3 \ldots}^{\infty} A_{r} \frac{2 a b(-1)^{(m+r-2) / 2} r m}{a^{2} r^{2}+b^{2} m^{2}}+\sum_{r=1,3 \ldots}^{\infty} B_{r} \frac{2 a b(-1)^{(m+r-2) / 2} r m}{a^{2} r^{2}+b^{2} m^{2}} \tanh \frac{r \pi a}{2 b} \\
& \quad-C_{m} \frac{a}{b}\left(\tanh \frac{m \pi b}{2 a}+\frac{\frac{m \pi b}{2 a}}{\cosh ^{2} \frac{m \pi b}{2 a}}\right)=0 .
\end{aligned}
$$

These equations are solved numerically by neglecting all terms higher than a given order, which results in a system of simultaneous equations.

The increasing usage of flat plates in the construction of panels in such steel structure as bridges and decks has called attention to the need for more information on the behaviour of rectangular plates with uniformly distributed loads. The center deflection of rectangular plates with clamped at four edges and subject to the action of uniformly distributed loads is an important problem that has received considerable attention because of its technical importance.

Substituting the values of the coefficients as given by equations (4), (5) and (6), inclusive, in equation (3), one obtains the center deflection of the rectangular plate, satisfying equation (1) and the boundary conditions, in the following form

$$
\begin{array}{r}
x=0 \\
y=0
\end{array}=\frac{q b^{4}}{24 D}\left\{1+\sum_{m=1,3 \ldots}^{\infty}\left[A_{m} \frac{1}{\cosh \frac{m \pi a}{2 b}}-C_{m} \frac{\sinh \frac{m \pi b}{2 a}}{\cosh ^{2} \frac{m \pi b}{2 a}}\right]\right\} .
$$

From the boundary condition, $B_{m}=0$, for the center deflection of the rectangular plate, it is clearly seen that there is no contribution of the coefficient $B_{m}$ on the deflection. The series in this expression converges rapidly, and sufficient accuracy is obtained by taking only the first term. When the aspect ratio goes to infinity, the center deflection tends to $0.00260417 q b^{4} / D$. This result is in agreement with Evans (1939) and Taylor \& Govindjee (2004).

\section{Square plate example and results}

Equations (4), (5) and (6) were solved for a square plate, $b / a=1$ using the first two terms of each series and resulted in the following values for the coefficients:

$$
\begin{aligned}
& A_{1}=-2.969687, \quad C_{1}=-0.585102 \\
& A_{3}=-0.7630, \quad C_{3}=0.2540
\end{aligned}
$$


Table 1. Numerical factors for center deflections for clamped square plate with uniform load.

\begin{tabular}{ll}
\hline Methods & $\begin{array}{c}\text { Numerical factor } \\
\alpha=\frac{w(0,0)}{q b^{4} / D}\end{array}$ \\
\hline Present work & $0 \cdot 00126401$ \\
(Timoshenko \& Woinowsky-Krieger, 1959) & $0 \cdot 00126$ \\
(Young, 1940) & $0 \cdot 00126$ \\
(Evans, 1939) & $0 \cdot 00126$ \\
(Wojtaszak, 1937) & $0 \cdot 0012637$ \\
\hline
\end{tabular}

It is seen that the second term of the series is negligible and by taking only the first term the formula for deflection is obtained.

The values of the deflections at the point $x=0, y=0$ of the clamped square plate with a uniform load are obtained from the method presented in this paper as well as those cited from Timoshenko \& Woinowsky-Krieger (1959), Young (1940), Evans (1939) and Wojtaszak (1937) are presented in table 1. In the calculations for the value of Poisson's ratio has been taken as $v=0 \cdot 3$. The results are in agreement with other studies.

\section{Conclusions}

This paper presents the center deflection of uniformly loaded isotropic rectangular plate with four clamped edges. The expansion of the center deflection function of the rectangular plate is in a series form and the second term of the series is negligible and that by taking only the first term the formula for deflection is obtained. The method presented in this paper is valid for most commonly used metal materials and gives a good approximation for most practical cases. A comparison with the previous studies shows that there is a reasonable agreement between the results.

The authors gratefully acknowledge the advice and encouragement of Professor M E Erdoğan in carrying out this work and would like to thank a referee for valuable comments.

\section{References}

Erdogan M E, Arpaci A 1990 On the deflections of uniformly loaded rectangular plates with clamped edges. Unpublished work

Evans T H 1939 Tables of moments and deflections for a rectangular plate fixed on all edges and carrying a uniformly distributed load. J. Appl. Mech. Trans. ASME 6: A7-A10

Hencky H 1913 Der Spannungszustand in rechteckigen Platten. Ph.D Thesis, Darmstadt, R. Oldenburg, Munich, Berlin

Hutchinson J R 1992 On the bending of rectangular plates with two opposite edges simply supported. J. Appl. Mech. Trans. ASME 59: 679-681

Imrak C E, Gerdemeli I 2007 A numerical method for clamped thin rectangular plates carrying a uniformly distributed load. Int. Appl. Mech. (accepted to publish) 
Szilard R 1974 Theory and analysis of plates (New Jersey: Prentice-Hall, Englewood Cliffs)

Taylor R L, Govindjee S 2004 Solution of clamped rectangular plate problems. Communi. Numer. Meth. Eng. 20: 757-765

Timoshenko S P 1938 Bending of rectangular plates with clamped edges. Proc. 5th Int. Congress of Applied Mechanics, (MIT: USA) 40-43

Timoshenko S P, Woinowsky-Krieger S 1959 Theory of plates and shells (New York: McGraw-Hill)

Timoshenko S P, Gere J M 1961 Theory of elastic stabilit. (New York: McGraw-Hill)

Wang C M, Wang Y C, Reddy J N 2002 Problems and remedy for the Ritz method in determining stress resultant of corner supported rectangular plates. Comput. Struct. 80: 145-154

Wang D, El-Sheikh A I 2005 Large deflection mathematical analysis of rectangular plates. J. Eng. Mech. 131: 809-821

Wojtaszak I A 1937 The calculation of maximum deflection, moments, and shear for uniformly loaded rectangular plate with clamped edge. J. Appl. Mech. Trans. ASME 4: A173-A176

Young D 1940 Analysis of clamped rectangular plates. J. Appl. Mech. 62: A139-A142 